\title{
BÚSQUEDA DE EXPEDIENTES DE FORMA VISUAL
}

\author{
Isabel Medrano-Corrales, Diego Ruiz-Macías y María-José Escalona-
}

Cuaresma

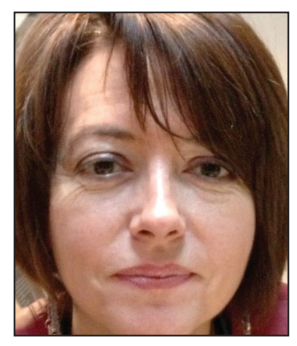

Isabel Medrano-Corrales es licenciada en geografía e historia por la Universidad de Sevilla. Desde 1998 es jefa de archivo y documentación de la Agencia de Obra Pública de la Junta de Andalucía, donde ha participado en el proceso de diseño e implantación del módulo de gestión documental integrado en un sistema de gestión empresarial. Continúa trabajando y estudiando la optimización de un enterprise resource planning (ERP) en un entorno de documento electrónico e interoperabilidad. Es profesora colaboradora del máster de documentación digital de la Universidad Pompeu Fabra. http://orcid.org/0000-0002-3419-9180

Agencia de Obra Pública de la Junta de Andalucía Av. Diego Martínez Barrio, 10. 41071 Sevilla, España isabel.medrano@aopandalucia.es

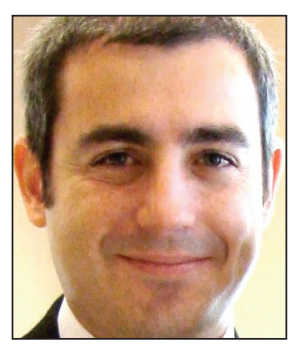

Diego Ruiz-Macías es ingeniero de telecomunicación, con intensificación de telemática por la Escuela Superior de Ingenieros de Sevilla. Es consultor senior en la empresa Tecnocom España Solutions desde 2007, especializado en proyectos de consultoría e implantación de sistemas de gestión documental y en el desarrollo de aplicaciones de gestión a media en tecnología Java. Anteriormente fue jefe de proyectos en la empresa Caymasa El Sendero especializado en proyectos basados en software libre con tecnología Java. Tiene especial interés en el uso de metodologías ágiles para el desarrollo de los proyectos.

http://orcid.org/0000-0002-2819-3970

Tecnocom España Solutions Av. de la Innovación, s/n. Edif. Rentasevilla pl. 5. 41020 Sevilla, España diego.ruiz@tecnocom.es

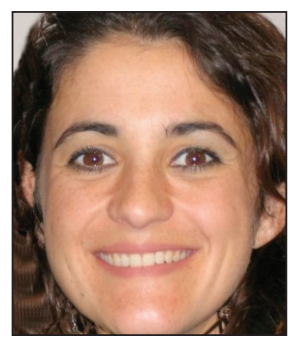

María-José Escalona-Cuaresma es ingeniera superior en informática por la Universidad de Sevilla donde consiguió su título de doctora europea en 2004. Es directora del Grupo de Investigación de Ingeniería Web y Testing Temprano y está adscrita al Departamento de Lenguajes y Sistemas Informáticos de la misma universidad donde trabaja como profesora titular. Como investigadora, sus líneas de trabajo están orientadas al campo de la ingeniería de requisitos y del aseguramiento de la calidad del software. Tiene una dilatada trayectoria en dirección de proyectos de investigación e investigación aplicada.

http://orcid.org/0000-0002-6435-1497

Universidad de Sevilla. ETS Ingeniería Informática Av. Reina Mercedes, s/n. 41012 Sevilla, España mjescalona@us.es

\section{Resumen}

Se muestran los resultados de la aplicación de técnicas de visualización de grandes conjuntos de documentos en el marco del Proyecto de innovación de la gestión documental aplicada a expedientes de contratación de servicios y obras de infraestructuras de transporte. En un entorno de documento electrónico, se parte de un esquema de metadatos como origen de la información que servirá para construir las visualizaciones. De los cuatro modos de visualización utilizados -timeline, cluster, nube de puntos y mapa- se describen con exhaustividad los metadatos empleados para cada uno de ellos y las representaciones visuales resultantes.

\section{Palabras clave}

Documentos de archivo, Expedientes de contratación, Archivos, Visualización, Búsqueda y recuperación de documentos, Líneas de tiempo, Nubes de puntos, Clusters, Mapas.

\section{Title: Searching records visually}

\begin{abstract}
We present the results of the application of visualization techniques to search large sets of records, under the framework of the "Project of innovation in record management applied to transport infrastructure services and works procurement dos-
\end{abstract}


siers". We started from a metadata schema as the origin of the information used to create visualizations. Four visualization techniques -timeline, cluster, cloud and map- and their metadata are exhaustively described.

\section{Keywords}

Records, Procurement dossiers, Archives, Visualization, Search and records retrieval, Timelines, Network diagrams, Clusters, Maps.

Medrano-Corrales, Isabel; Ruiz-Macías, Diego; Escalona-Cuaresma, María-José (2014). “Búsqueda de expedientes de forma visual". El profesional de la información, mayo-junio, v. 23, n. 3, pp. 330-338.

\section{Introducción}

La búsqueda de documentos de archivo en un entorno electrónico conlleva el diseño de estrategias para traducir nuestra necesidad de información a las posibilidades de una interfaz de búsqueda más o menos avanzada (Marcos, 2004; Red.es, 2013). Cuando se trata de realizar búsquedas difusas en un buscador simple, nuestras expectativas de éxito rápido dependerán de las posibilidades de filtrado posterior que ofrezca el sistema. En los casos de búsquedas de fondos, divisiones de fondos y series documentales, las herramientas de búsqueda aún se vuelven más estrictas que si se trata de documentos.

Los referentes visuales que siempre hemos tenido en un entorno papel y nos han servido para orientarnos, se han desvanecido

Los referentes visuales que siempre hemos tenido en un entorno papel y nos han servido para orientarnos, se han desvanecido. Por eso consideramos interesante explorar nuevas formas de visualización. Reconocemos como pionera en España la iniciativa del Archivo de la Ciudad de Arganda del Rey, que ofrece las opciones de visualizar los documentos que resultan de una búsqueda en una línea del tiempo y un mapa.

http://archivo.ayto-arganda.es/Búsquedas.aspx

En el Proyecto de innovación de la gestión documental aplicada a expedientes de contratación de servicios y obras de infraestructuras de transporte ${ }^{1}$, se incluye el empleo de técnicas de visualización de grandes conjuntos de datos a la localización de documentos. Los resultados de este proyecto -aún en fase de ejecución- se han diseñado para poder reutilizarse por cualquier órgano contratante de la Junta de Andalucía y, por extensión, por cualquier administración con competencias en materia de contratación de servicios y obras (Dietrich; Myrttinen, 2012). El proceso de adopción de los modelos y herramientas de software por parte de cualquier organismo interesado requerirá previa o simultáneamente dotarse de un nivel de identificación, valoración y descripción documental cualificada. Es decir, deberá contarse con los estudios de identificación y valoración de las series documentales de expedientes de contratación de servicios y obras, así como la descripción de dichos expedientes y los documentos que contienen. El tratamiento archivístico es, si cabe, aún más imprescindible en un entorno electrónico.

Las visualizaciones se diseñaron en un principio para representar documentos -simples o expedientes-, pero durante el desarrollo de los trabajos se vio la oportunidad de aplicarlas también para visualizar relaciones entre otros niveles de agrupación: grupos de fondos, fondos, divisiones de fondos y series documentales.

\section{2. ¿Qué entendemos por "forma visual"?}

Entendemos por búsqueda y recuperación de documentos de "forma visual" la asistencia que se puede recibir de representaciones visuales que ayudan a comprender el volumen de información que se muestra y, además, sirven de orientación para afinar la búsqueda mediante la interacción con los elementos mostrados en la visualización.

Los objetivos generales que se pretenden alcanzar son:

- Facilitar la comprensión por los usuarios de grandes conjuntos de documentos de archivo; una práctica habitual en el caso de los documentos bibliográficos, para los que existe una tradición de estudios bibliométricos y de visualización. Ligado a ello se pretende intensificar la orientación al usuario, también adoptando maneras de hacer ya consolidadas en el ámbito bibliotecario (Bengochea; Patricio, 2005).

- Reducir el tiempo de búsqueda, ofreciendo al usuario opciones intuitivas con referentes visuales que le asistan.

- Visualizar la información contextual de los documentos contenida en metadatos; por ejemplo, visualizar los documentos en el tiempo, en el espacio, en los hitos de la tramitación administrativa y en sus relaciones con otros documentos.

- Evidenciar visualmente relaciones que habitualmente permanecen ocultas o escondidas entre las categorías de la entidad documento y que son muy útiles en la búsqueda de información; por ejemplo, visualizar las relaciones de un expediente con otros o de una serie documental con otras es algo que en un entorno papel es información registrada en un formulario o informe de uso casi exclusivamente interno.

Adicionalmente identificamos otros objetivos específicos en relación a la contratación de obras y servicios de transporte:

- Facilitar las búsquedas de documentos que componen los expedientes de contratación de obras y servicios de infraestructuras del transporte, que se caracterizan por ser voluminosos -por su gran número de documentos-, exten- 
sos -sobre todo las obras de infraestructuras se prolongan en el tiempo hasta su recepción definitiva- y complejos al contener documentos que en sí son objetos complejos, como los proyectos de construcción-.

- Presentar visualizaciones de los documentos orientadas al usuario, para lo cual se emplean los referentes que se conocen como más intuitivos para esta tipología documental: espacio (situación geográfica del objeto de cada expediente) (Bosque-Sendra; Zamora-Ludovic, 2002), tiempo (localización temporal de cada documento) y génesis (relación con la tarea que lo genera).

- Ofrecer posibilidades de interacción en las visualizaciones de documentos para que los usuarios puedan adaptar determinados parámetros de la representación de manera personalizada. Para ello previamente es precisa una labor de identificación de los metadatos que intervienen en estos procesos de interacción.

El principal obstáculo para la consecución de los objetivos anteriores es el riesgo de deficiencias en la calidad de los metadatos, que provoquen una extracción incierta y una visualización incorrecta.

Debemos explorar nuevas formas de visualización para hacer más comprensible la realidad de los documentos en el entorno electrónico

\section{El origen de los datos para la visualización}

La naturaleza y estado de los datos sobre los documentos objeto de visualización son una cuestión sustancial en el proceso. En un entorno electrónico los datos sobre los documentos son los metadatos y requieren estar definidos en un perfil de aplicación de un modelo de metadatos (Chen, 1999). Esta circunstancia propicia que la información estructurada pueda ser seleccionada, tratada y explotada mediante representaciones visuales de datos.

En el caso que nos ocupa, el perfil de metadatos sigue el Esquema de metadatos para la gestión del documento electrónico (e-Emgde ${ }^{2}$ ) propuesto por el Ministerio de Hacienda y Administración Pública en el marco de aplicación de la Norma técnica de interoperabilidad de política de gestión de documentos electrónicos.

Se ha optado por un modelo multientidad con cinco entidades:

- documento;

- agente;

- actividad;

- regulación;

- relación.

De todas ellas, será la entidad documento la que suministre la mayoría de la información que servirá para elaborar las visualizaciones. Dentro ella, se distinguen varias categorías con el objetivo de crear una estructura jerárquica documental: grupo de fondos, fondo, división de fondo, serie documental, expediente, documento.
El lugar natural donde se implementa el perfil de metadatos es el gestor documental. En él se debe situar toda la lógica de la información sobre los documentos electrónicos. De forma complementaria, se podrán desarrollar servicios que optimicen la extracción de datos para la visualización de los documentos. Esta ha sido la solución empleada en el proyecto que exponemos.

En lo que respecta a las visualizaciones, se han considerado cuatro tipos: timeline, clúster, nube de puntos y mapa.

A modo de esquema, en la figura 1 se muestran los metadatos ofrecidos por la entidad documento, en cada una de sus categorías, y que son utilizados por las visualizaciones. A continuación se analiza de dónde procede la información empleada en los distintos tipos de visualización propuestos:

\subsection{Vista timeline}

Muestra los documentos de un expediente situados a lo largo de una línea de tiempo, y las fases y tareas realizadas en su tramitación. Es por ello que en esta visión están involucradas la entidad "documento" -y en concreto, sus categorías "expediente" y "documento simple"- y la entidad "actividad". La información utilizada para construir esta vista se obtiene de:

\section{Entidad "documento"}

a) Categoría "expediente":

- El nombre del expediente <Nombre.NombreNatural> figura en la pantalla inicial de la visualización.

- La descripción del expediente <Descripción>.

b) Categoría "documento simple":

- El nombre de cada documento limitado a 40 caracteres $<$ Nombre.NombreNatural> se presenta en una viñeta en la barra temporal. Si seleccionamos esta viñeta, visualizamos en la parte superior el nombre completo del documento.

- La descripción <Descripción>.

- La fecha de captura <Fecha.Fechalnicio>, que refleja la fecha de creación del documento en el sistema.

- Metadatos específicos que el usuario puede elegir para modificar la visualización, por ejemplo, otras fechas del documento relativas a su tramitación y que hacen que cambie la ubicación de cada uno en el timeline <Fecha. FechaDocumento>; o la valoración o no de un documento como esencial, criterio que reduce la visualización del expediente a sus documentos esenciales.

\section{Entidad "actividad"}

a) Las categorías "fase" y "tarea" están relacionadas con los tipos de documentos identificados en el procedimiento de contratación:

- De esta forma, el nombre de la fase o de la tarea <Nombre.NombreNatural> nos ayuda -junto con la fecha del documento- a contextualizar el documento.

\subsection{Vista clúster}

Modo de visualización que el usuario puede elegir para tener una visión de las relaciones de asociación que existen entre los nodos del cuadro de clasificación de documentos 
(Fernández-Molina; Peis, 1999), hasta el nivel de serie documental. Para construir esta representación se utiliza información procedente de:

\section{Entidad "relación", categoría "relación de procedencia"}

a) El metadato <TipoRelación>, que expresa como uno de los valores el tipo de relación de procedencia asociativo, que es el que nos interesa.

b) El número de relaciones de asociación, cuyo cómputo se emplea para aproximar los nodos con más relaciones y viceversa.

c) El número de relaciones de tipo "contiene" entre un elemento y sus hijos, para ajustar el tamaño de cada nodo al volumen de objetos existentes. Por ejemplo, los nodos que representan cada serie documental tienen el tamaño proporcional al número de expedientes de esa serie.

\section{Entidad "documento"}

a) Categorías "fondo", "división de fondo" y "serie documental":

- Nombre <Nombre.NombreNatural> del fondo, división de fondo o serie documental, que se presenta como etiqueta sobre su punto correspondiente en la nube.

- Descripción <Descripción> del fondo, división de fondo o serie documental que hayamos seleccionado y cuyo texto visualizamos en la parte inferior de la pantalla.

- Otros metadatos específicos que se hayan configurado en la pantalla de preferencias para que aparezcan como información de detalle del objeto seleccionado en la parte inferior de la pantalla.

\subsection{Vista nube de puntos}

Modo de visualizar un expediente mostrando su diagrama de relaciones -de subordinación o asociación- con otros expedientes. Obtiene la información de la entidad "documento" y, en concreto, de las categorías "expediente" y "documento simple", ya que la nube de puntos mostrará solamente las relaciones entre los elementos de estas categorías:

- Nombre del expediente o documento < Nombre.NombreNatural>.

- Descripción del expediente o documento <Descripción>.

- Otros metadatos específicos que hayamos configurado en nuestra pantalla de preferencias para que nos aparezcan como información de detalle del objeto seleccionado, en la parte inferior de la pantalla.

\subsection{Vista mapa}

Se posicionan los expedientes en un sistema de cartografía. La información se extrae de la entidad "documento" y, en concreto, de la categoría "expediente":

- Las coordenadas del objeto del expediente -es decir, las del proyecto u obra de infraestructura- se obtienen de los metadatos <PuntoAcceso.Lugar.Coordenadas.Latitud> y $<$ PuntoAcceso.Lugar.Coordenadas.Longitud $>$.

- El nombre se obtiene del metadato <Nombre.NombreNatural> y se visualiza en un pop-up sobre el punto correspondiente en el mapa
- La descripción se obtiene del metadato <Descripción>.

- La información del pop-up puede completarse con otros metadatos específicos que se hayan configurado en la pantalla de preferencias para que aparezcan como información de detalle del objeto seleccionado.

Hay que ofrecer al usuario opciones más intuitivas para buscar documentos, en las que encuentre referentes visuales que le asistan

\section{Tipos de visualizaciones de conjuntos de documentos de archivo según el modelo de grafo}

A continuación se describen los cuatro tipos de visualizaciones utilizadas y se exponen los requisitos de uso:

\subsection{Timeline}

\section{- Propósito}

El objetivo es ofrecer una panorámica visual de los documentos que contiene un expediente, con dos referencias en la banda inferior:

- la línea de tiempo que marca la fecha del documento

- el nombre de la tarea que da origen al documento.

Es una alternativa a las habituales interfaces tabulares que presentan una lista de documentos, distribuida en pantallas consecutivas por las que hay que navegar. Para el caso de expedientes voluminosos y largos como los de contratación de obras y servicios de infraestructuras, es un intento de hacer más accesible la complejidad de este tipo documental.

El usuario puede moverse con rapidez en el expediente cronológicamente, ampliar períodos concretos para ver con más detalle las viñetas de documentos y visualizarlos en la parte superior cuando marque la viñeta que le interese.

Este modo de visualización puede resultar especialmente útil a usuarios que no vayan buscando una información concreta, sino que quieran ver el panorama global de un contrato para luego navegar de forma más específica según las opciones que se vayan presentando. No obstante, si el usuario personaliza la visualización mediante el menú de sus preferencias, puede lograr un resultado mucho más afinado, por ejemplo, puede ver solamente los documentos seleccionados como "esenciales" para auditoría.

Los destinatarios de este tipo de visualización pueden ser gestores, auditores e incluso el público en general si se produce la difusión web en abierto. Según el perfil del usuario, tendrá permiso para ver todos o determinados documentos.

\section{- Aspectos visuales}

En el diseño de pantalla predomina el blanco y otros colores suaves que aportan un aspecto limpio y resaltan la información que se quiere mostrar. No se usan motivos de decoración para no restar protagonismo a los elementos informativos. 


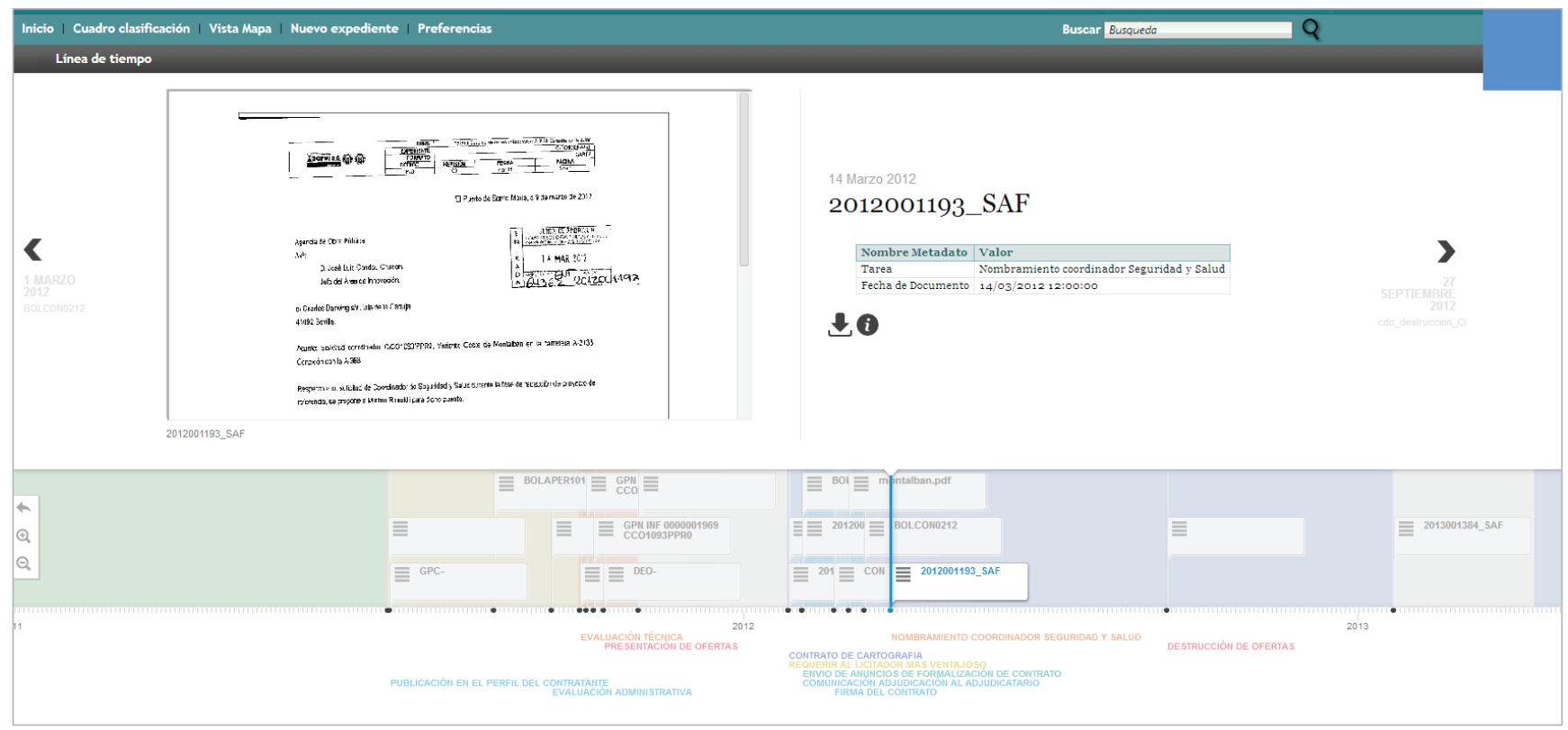

Figura 1. Visualización de un expediente en timeline

La interfaz está dividida en tres bandas horizontales: en la inferior se sitúa la línea de tiempo y el detalle de las tareas asociadas al cronograma, en la siguiente están las viñetas de los documentos sobre una franja de colores que representan la tarea que origina dichos documentos, y en la superior se visualiza la imagen del documento seleccionado en el timeline y un detalle del mismo.

La escala de tiempo se ajusta automáticamente en base a la información contenida en el expediente, o sea, al número de documentos, de forma que la visualización inicial del mismo resulte lo más compacta posible. Mediante una etiqueta flotante se accede al menú contextual, que permite cambiar a otro tipo de vistas del expediente: tabular, árbol, sólo documentos esenciales o todos, y establecer el tipo de fecha por la que se quieren visualizar los documentos en el timeline.

Cada icono sin leyenda tiene su descripción emergente; es el caso de los controles de la línea de tiempo -aumentar, disminuir zoom y volver al inicio de la línea del tiempo- y los iconos de "detalle del documento" y "descarga" referidos al documento que se ve en pantalla. Se han elegido letras claras y fuentes de una única familia; según la configuración del equipo será trebuchet ms, arial, verdana o helvética.

\section{- Funciones}

La función principal consiste en visualizar de forma gráfica y asociada a una línea temporal y a tareas de tramitación, los documentos de un expediente en una sola búsqueda, pudiendo el usuario desplazarse de dos formas: eligiendo pasar a ver el próximo/anterior documento mediante las flechas situadas a mitad de pantalla, o desplazando el cursor o rueda del ratón sobre la línea de tiempo. Las funciones del timeline son:

- aumentar/disminuir la escala;

- volver al inicio de la línea de tiempo;

- seleccionar un documento en el timeline.
Al seleccionar un documento, en el marco superior se muestra la imagen de la página primera y una ficha con metadatos mínimos descriptivos, pudiendo descargar el documento o acceder al registro descriptivo completo del documento.

La vista de timeline es útil a usuarios que no buscan una información concreta, sino el panorama global de un contrato para luego navegar de forma más específica

\section{- Interacción}

Para modificar la visualización, el usuario puede configurar sus preferencias a través del menú contextual que se le ofrece en una ventana flotante. Por ejemplo los metadatos que quiere ver en la ficha descriptiva del documento, el tipo de fecha de los documentos o si los quiere ver todos o una selección de los "esenciales".

\section{- Aplicaciones, componentes y servicios utilizados}

Esta vista se ha creado con TimelineJS, un componente Javascript para construir líneas de tiempo basadas en eventos asociándoles elementos multimedia. Permite integración directa con los servicios de Twitter, Flickr, Google Maps, YouTube, Vimeo, Vine, Dailymotion, Wikipedia, SoundCloud. Este componente ha sido modificado para facilitar funciones adicionales así como para realizar su integración en el sistema final.

http://timeline.knightlab.com

\subsection{Clúster}

\section{- Propósito}

Se pretende aproximar al usuario a las relaciones entre fondos, entre divisiones de fondos y entre series documenta- 
les, una información que tradicionalmente ha estado restringida a la consulta de los estudios de identificación de series documentales, que por otra parte no suelen tener una difusión muy abierta. En cambio sí que contienen información de utilidad tanto para los profesionales como para los usuarios.

\section{- Aspectos visuales}

En el diagrama (figura 2) se visualiza la entidad seleccionada -en distinto color-y sus relaciones de asociación con otros nodos. El tamaño de los nodos es proporcional al número de expedientes que contiene cada serie documental y la distancia entre nodos revela la intensidad de la relación, o sea, el número de relaciones entre los nodos. En este caso las series documentales de expedientes de contratación de obras y de servicios son las más voluminosas y las que más estrecha relación tienen. Es totalmente lógico, ya que la redacción de un proyecto (contrato de servicio) está directamente vinculada a la realización de la obra (contrato de obra) y a la dirección de obra (contrato de servicio).

Los nodos presentan el rótulo con su nombre y están unidos por líneas de igual grosor. El marco de la parte inferior muestra los metadatos básicos del objeto seleccionado, que puede configurar el usuario en su pantalla de preferencias.

Podemos visualizar los documentos en el tiempo, en el espacio, en los hitos de la tramitación administrativa y en sus relaciones con otros documentos

\section{- Funciones}

Esta vista propicia una visión contextualizada de las agrupaciones de documentos superiores al nivel de expediente. Eligiendo una de estas agrupaciones -fondo, división de fondo o serie documental- se representa el diagrama de relaciones de asociación con otras entidades.

\section{- Interacción}

El usuario puede navegar a través de los nodos y la visualización va cambiando, cargándose las relaciones de asociación del nuevo nodo con otros.

\section{- Aplicaciones, componentes y servicios utilizados}

Las vistas de nube de puntos y clúster basan su funcionamiento en el componente JSF mindmap de PrimeFace, al que se le ha ampliado su función básica con el desarrollo de clúster.

\subsection{Nube de puntos}

\section{- Propósito}

El objetivo de esta vista es que sean evidentes para el usua- rio las relaciones de "contiene" y de "asociación" entre expedientes, y las relaciones de "asociación" entre documentos. Un expediente según esta vista aparecerá enlazado a otros expedientes subordinados (relación de "contiene") y a otros expedientes relacionados (relación de "asociación"). Es una visualización sencilla y útil para los gestores, profesionales de la información y usuarios.

\section{La vista de clúster muestra una visión contextualizada de las agrupaciones de documentos superiores al nivel de expe- diente}

Se diferencia del clúster en que aquí la distancia entre nodos no tiene significado, mientras que el clúster aproxima los elementos según la intensidad de su relación.

\section{- Aspectos visuales}

El resultado es un diagrama de nodos unidos por líneas. En el centro está el objeto seleccionado y a su alrededor los demás objetos con los que se relaciona. El nodo principal figura en el centro en color destacado y los nodos relacionados en dos colores distintos, según estén unidos por relación de "asociación" o "contiene".

Los nodos tienen etiquetas con su nombre limitado a un número de caracteres, ya que los nombres de los expedientes o documentos pueden ser bastante largos. Al situar el cursor sobre un nodo, se visualiza una etiqueta emergente con el nombre completo. Las líneas de relación son iguales en todos los casos, tanto en grosor como en distancia. El marco de la parte inferior muestra los metadatos básicos del objeto seleccionado, que puede personalizar el usuario. 


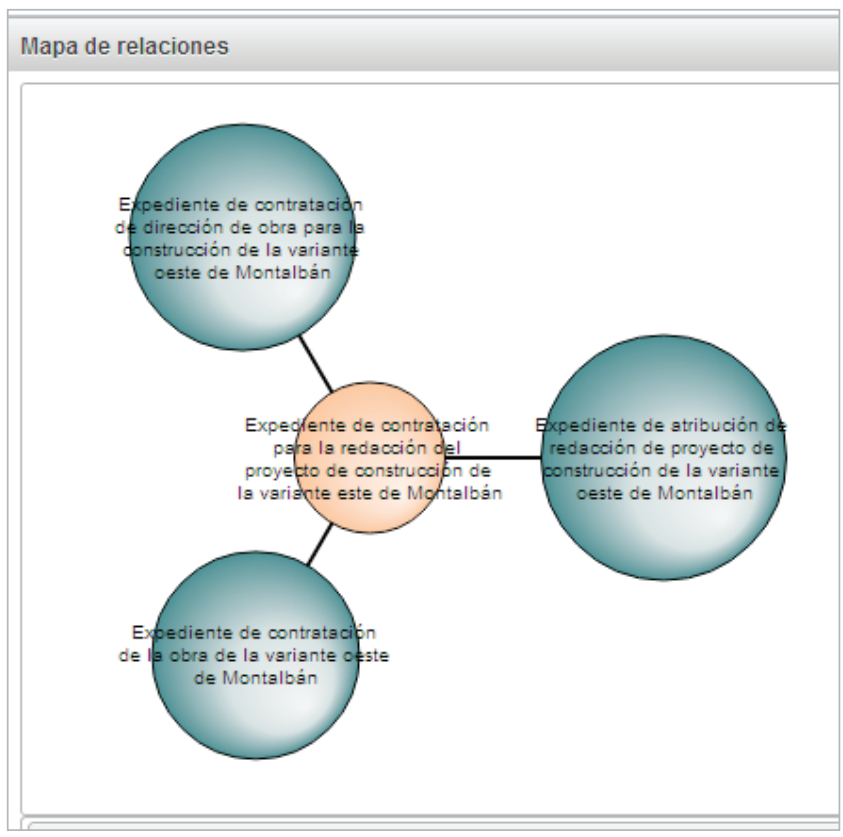

Figura 3. Visualización de la relación de un expediente con otros

\section{- Funciones principales}

Proporciona una visión contextualizada de documentos y expedientes, al mostrar sus relaciones de "asociación" o "contiene".

\section{- Interacción}

El usuario puede modificar la visualización organizando los nodos para mejorar la composición del diagrama. En el caso del clúster, los nodos no se podían mover porque la distancia estaba fijada en función de la intensidad de la relación entre nodos. También se pueden seleccionar otros nodos y se cargan las relaciones de ese objeto con otros.

La vista de nube de puntos proporciona una visión contextualizada de documentos y expedientes

\subsection{Vista mapa}

\section{- Propósito}

Cuando el objeto de los contratos tiene un alto significado geográfico -como es el caso de los contratos de obras y servicios de infraestructuras de transporte- la visualización de los expedientes de contratación posicionados en un mapa resulta una forma muy intuitiva de ofrecer la información al usuario.

Esta vista es una opción que tiene el usuario en la pantalla de resultados, tras realizar una búsqueda. El propio mapa puede convertirse en una prolongación de la búsqueda si el usuario interacciona y llega a los documentos a través de los iconos que los señalan.

\section{- Aspectos visuales}

En la pantalla inicial se muestra la cartografía centrada en Andalucía porque es el territorio donde se sitúan los docu- mentos tratados. Los expedientes se visualizan mediante puntos que, si están muy cercanos y la escala es grande, se agrupan en puntos mayores y proporcionales al número de documentos. A medida que se aumenta el zoom se van individualizando los puntos.

\section{- Funciones}

Esta vista proporciona de forma gráfica la ubicación de los expedientes en una posición geográfica y facilita al usuario el análisis de la información que visualiza, a la vez que proporciona asistencia si quiere seguir la búsqueda y su referente es el espacio.

\section{- Interacción}

El mapa permite realizar zoom más/menos. Al colocar el cursor sobre un punto emerge el nombre del expediente y al hacer clic se verán sus metadatos principales y se podrá acceder directamente al mismo. Estos metadatos son configurables por el usuario en su perfil de preferencias.

\section{- Aplicaciones, componentes y servicios utilizados}

La vista mapa basa su funcionamiento en los siguientes componentes:

- OpenStreetMap: servicio de cartografía bajo licencia abierta (con licencia Creative Commons ReconocimientoCompartirlgual 2.0 (CC BY-SA)) y creado por las aportaciones de su comunidad de usuarios. http://www.openstreetmap.org

- Leaflet: librería JavaScript open source que permite añadir capas de forma sencilla a una cartografía. http://leafletjs.com

- MarkerClusterer plugin para Leaflet: librería open source JavaScript que permite la creación de clústeres de puntos de interés en una cartografía, de forma que los puntos de interés se agrupan o se separan en función del zoom aplicado

https://github.com/Leaflet/Leaflet.markercluster

\section{La vista de mapa muestra la ubicación de los expedientes en una posición geográ- fica y facilita al usuario el análisis de la información que visualiza}

\subsection{Requisitos de uso}

Los mecanismos de visualización descritos se encuadran en una aplicación web desarrollada con tecnología Java y basada en los siguientes frameworks:

- JSF 2.0 con implementación Mojarra 2.1.2 y librerías de componentes Jboss RichFaces y PrimeFaces.

http://www.jboss.org/richfaces

http://www.primefaces.org

- Spring Framework 3.1.2 con Spring Security para la gestión de la seguridad.

- Estándar de servicios web JAX-WS con la implementación de Apache CXF 2.7.7. 
Los requisitos mínimos que debe cumplir el servidor en el que se instale la aplicación son:

- Máquina virtual Java 1.6 o superior.

- Servidor de aplicaciones Jboss 7.1 con implementación de servicios JBossWSCXF-4.1.1.Final.

A nivel de usuario de la aplicación, los requisitos que debe disponer para usar los mecanismos de visualización son:

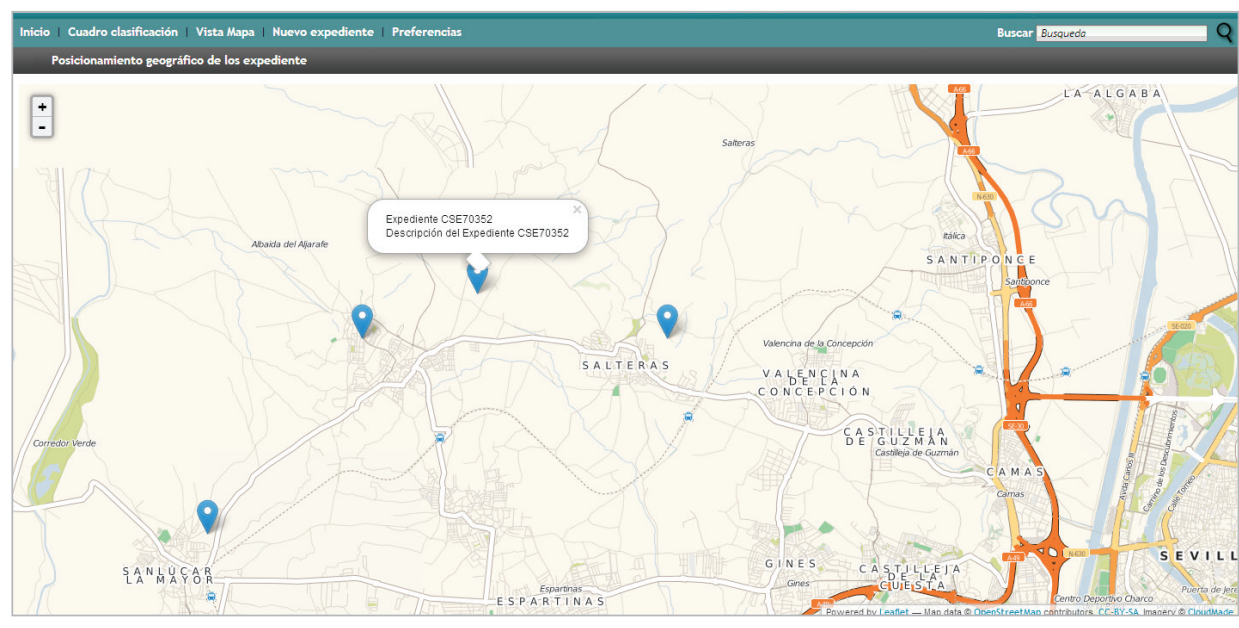

Figura 4. Visualización en mapa de expedientes de contratación
- Navegador web Internet Explorer 9 o superior, Google Chrome en su última versión o Mozilla Firefox en su última versión.

- Resolución de pantalla mínima de 1.024 x 768 y resolución recomendada de 1.920 x 1.080 .

\section{Conclusiones}

Es evidente que los documentos de archivo son una fuente de información única. Pero ganarían mucho los usuarios si utilizamos todos los medios a nuestro alcance para facilitarles el contacto con los documentos. La orientación al usuario es la máxima que debe inspirar la labor de difusión de los documentos de archivo.

Este es el motivo que nos ha llevado a emplear técnicas de visualización para hacer más comprensivo y directo el conocimiento (Dürsteler, 2002; Costa, 1998) de un tipo de expedientes complejo por la cantidad de documentos que contienen y por la extensa tramitación en el tiempo: los expedientes de contratación de servicios y obras de infraestructuras de transporte. Estos expedientes se podrán consultar asociados a su localización geográfica o a través de las relaciones -ya sea de pertenencia o de asociación- con otros expedientes. Una vez dentro del expediente, se podrán visualizar los documentos que lo componen, asociados a una línea de tiempo o de forma tabular inscritos en la fase y tarea del procedimiento que le corresponda a cada uno.

Con el objetivo de optimizar estas visualizaciones y extender su aplicación a más conjuntos de documentos, las herramientas han sido parametrizadas para que puedan representar también otro tipo de expedientes, así como series documentales, divisiones de fondos y fondos. Todos estos elementos se pretenden incluir en un intento de ofrecer visualmente al usuario un volumen de información estructurada pero de difícil comprensión.

Las visualizaciones son consecuencia de la gestión de los metadatos de los documentos, cuyos valores contienen los datos que precisamos para producirlas. De la calidad de estos datos estructurados dependerá el éxito y la certeza de las visualizaciones que obtengamos.

La experiencia que se expone en este artículo no es original en su desarrollo, pero sí en su aplicación en un ámbito falto de estímulos para buscar documentos de forma visual. Se- ría deseable que surgiesen más iniciativas en este sentido, aprovechando la oportunidad de tratamiento que nos ofrece la información altamente estructurada propia del entorno del documento electrónico.

\section{Reconocimientos}

Los autores agradecen la financiación de la Unión Europea al Proyecto de innovación de la gestión documental aplicada a expedientes de contratación de servicios y obras de infraestructuras de transporte a través del Programa Operativo Feder de Andalucía 2007-2013. También agradecen su labor a los miembros del equipo de investigación de Ingeniería Web y Testing Temprano de la Universidad de SeviIla, a la empresa Tecnocom España Solutions, así como a los directivos e investigadores de la Agencia de Obra Pública de la Junta de Andalucía, por su dedicación y profesionalidad.

Las técnicas de visualización hacen más comprensivo y directo el conocimiento de un expediente complejo

\section{Notas}

1. Uno de los 28 proyectos de $\mathrm{I}+\mathrm{D}+\mathrm{i}$ relacionados con los ámbitos y competencias de la Consejería de Fomento y Vivienda, que fueron licitados y contratados en 2012 por la Agencia de Obra Pública de la Junta de Andalucía, todos cofinanciados por la Unión Europea (Programa Operativo Feder de Andalucía 2007-2013). Este en concreto fue adjudicado al Grupo de investigación de Ingeniería Web y Testing Temprano de la Universidad de Sevilla, continúa en ejecución y está prevista su finalización para junio de 2014.

2. Esquema de metadatos para la gestión del documento electrónico (e-Emgde). Documentación complementaria a la Norma técnica de interoperabilidad de política de gestión de documentos electrónicos. Ministerio de Hacienda y Administraciones Públicas.

\section{Bibliografía}

Bengochea, Luis; Patricio, Miguel-Ángel (2005). "Sistemas de visualización para bibliotecas digitales". Revista española 
de documentación científica, v. 28, n. 3, pp. 273-292.

http://redc.revistas.csic.es/index.php/redc/article/ view/170/224

http://dx.doi.org/10.3989/redc.2005.v28.i3.170

Bosque-Sendra, Joaquín; Zamora-Ludovic, Hernán-Eduardo (2002). "Visualización geográfica y nuevas cartografías". Geofocus: revista internacional de ciencia y tecnología de la información geográfica, n. 2, pp. 61-77.

http://geofocus.rediris.es/docPDF/Articulo4_2002.pdf

Costa, Joan (1998). Información visual y conocimiento. La esquemática. Visualizar la información. Barcelona: Paidos. ISBN: 9788449306112

Chen, Chaomei (1999). Information visualization and virtual environments. London: Springer. ISBN: 1852331364

Dietrich, Daniel; Myrttinen, Henri (2012). Data visualization. European Public Sector Information Platform.

http://www.epsiplatform.eu/sites/default/files/Final\%20 TR\%20Data\%20Visualisation.pdf

Dürsteler, Juan-Carlos (2002). Visualización de la información: proceso de interiorización del conocimiento mediante la percepción de información.

http://www.infovis.net/printMag.php?num=100\&lang=1

Fernández-Molina, Juan-Carlos; Peis, Eduardo; (1999). "Uso de esquemas de clasificación para mejorar las prestaciones de visualización (browsing) de los catálogos en línea". En: Organización del conocimiento en sistemas de información y documentación: III Encuentro de ISKO-España, Getafe, 19-21 nov. 1997, pp. 295-306.

http://dialnet.unirioja.es/descarga/articulo/2036344.pdf

Inf@Vis! La revista digital de InfoVis.net.

http://www.infovis.net

Marcos, Mari-Carmen (2004). Interacción en interfaces de recuperación de información: conceptos, metáforas y visualización. Gijón (Asturias): Trea. ISBN: 8497041186

Red.es (2013). Recopilación de herramientas de procesamiento y visualización de datos. Madrid

http://datos.gob.es/sites/default/files/Herramientas_de_ Visualizaci\%C3\%B3n_vf.docx

\section{Directorio EXIT \\ (EXpertos en Tratamiento de la Información) http://directorioexit.info}

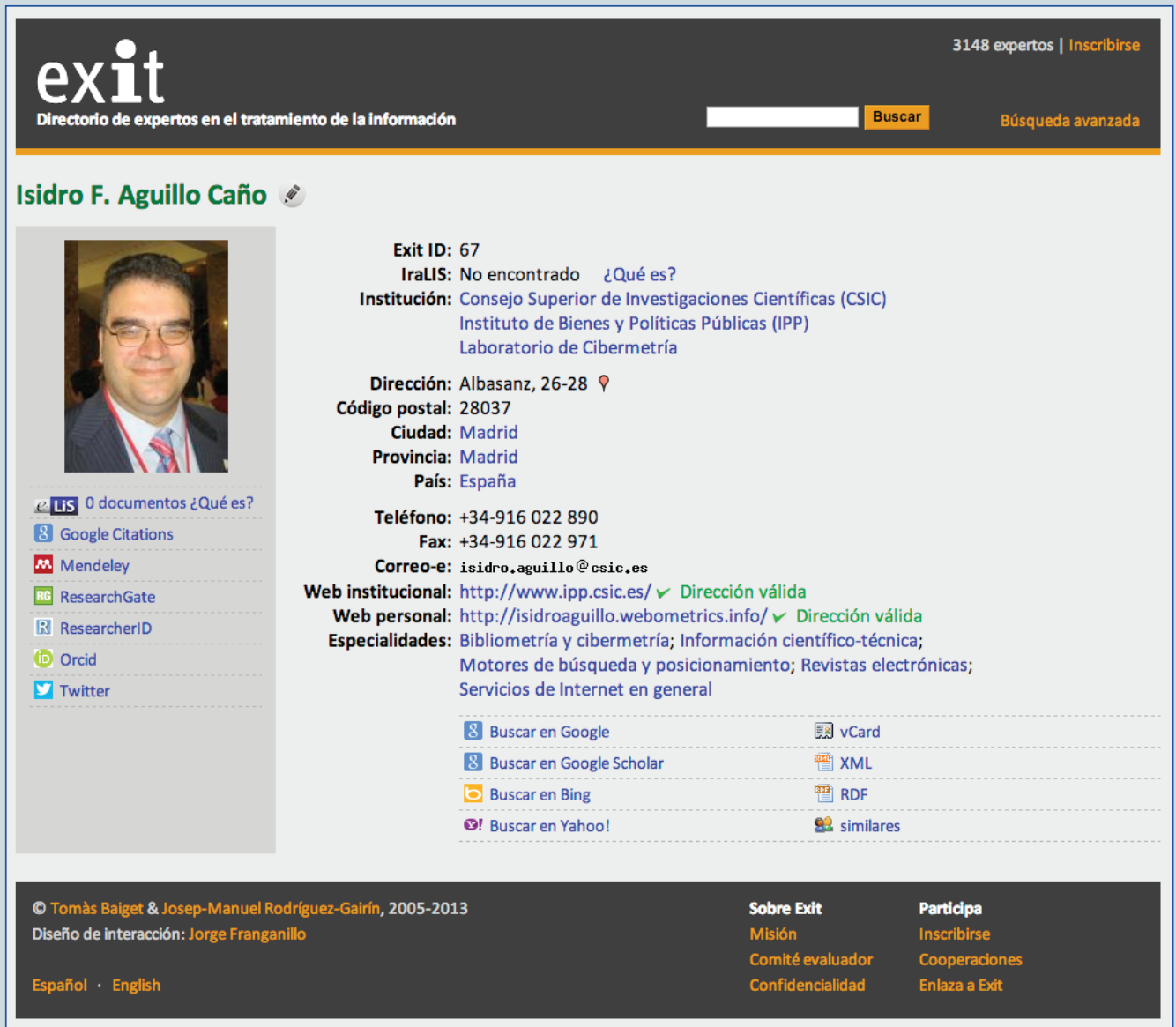

Una ventana a las oportunidades profesionales 